
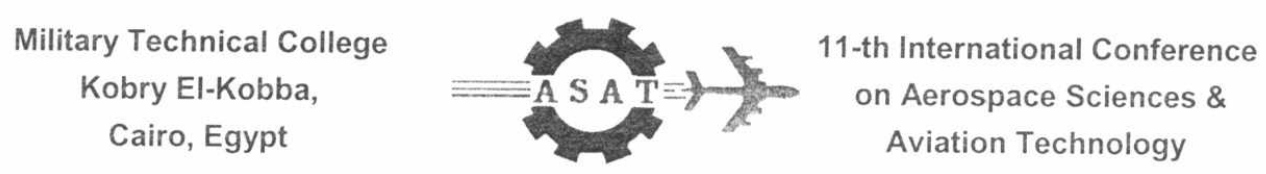

\title{
EFFECT OF IMPERFECT POWER CONTROL ON PARALLEL INTERFERENCE CANCELLATION FOR CDMA
}

\author{
Yahya Mohasseb* and Hisham Elgamal*
}

\begin{abstract}
This paper presents a study of the effect of imperfect power control on the performance of a Partial Parallel Interference Cancellation (PPIC) multiuser receiver. We show that the PPIC receiver can absorb the effect of imperfect power control to a great extent compared with other types of receivers. Additionally, the effect of imperfect power control on the optimum weights of the PPIC receiver is considered. It is found that imperfect power control causes the receiver to become more susceptible to performance degradation as a result of deviation from optimal weights.
\end{abstract}

\section{KEYWORDS}

CDMA, Parallel Interference Cancellation (PIC), Partial Parallel Interference Cancellation (PPIC), Power Control in CDMA.

\section{INTRODUCTION}

The performance of a Code Division Multiple Access (CDMA) system is limited by multiple access interference (MAI). Such a problem arises from the use of the conventional single user detector, which ignores the existence of other users. As a consequence, when the number of active users increase to a certain level or when some users signals become extremely strong, weak users with the conventional single user detection may lose communication because of the overwhelming MAI [1].

\footnotetext{
Egyptian Armed Forces

". Port Said Faculty of Engineering, Suez Canal University
} 
This is known as the near-far problem. This problem is better solved using multiuser detection [2] Various optimal and suboptimal multiuser detectors have been proposed. The optimal detector [3] can significantly eliminate the MAI, however its complexity exponentially increases with the number of users and the length of the sequence [4]. Such complexity prohibits practical implementation. Suboptimal echniques can be classified into two categories: Linear multiuser detectors \& nonlinear multiuser detectors (subtractive interference canceller). In linear multiuser detection, a linear transformation is applied to the soft output of the conventional detector (Matched Filter) in order to eliminate the MAI. An example of linear suboptimal detector is the decorrelator detector [5]. In non linear detectors, we use subtractive interference cancellation where the interference is estimated and removed from the received signal before detection. This removal could be either successively [6] or in parallel [7], [8]. However, parallel interference cancellation (PIC) causes much less decision delay than the successive scheme.

PIC can be implemented in multiple stages to improve performance. A correct estimate of a certain interfering user's bit lead to a successful removal of interference. However, if a wrong decision is subtracted, the increased interference power would be fourfold as high as compared to those without any cancellation [1]. Divsalar \& Simon [4] suggested a Partial Parallel Interference Cancellation (PPIC) of the MAl by introducing a weight in each stage to determine the amount of cancellation. In adaptive PPIC, the Least Mean Square (LMS) algorithm is employed to obtain the optimal weights [1], but at the cost of more complexity compared with both the PIC and the PPIC

Power control is one of the most important system requirement in DS-CDMA systems and received wide attention in academic literature. If power control is not used all mobiles transmit signals toward the base station with the same power without taking into account the fading and the distance from the base station. Mobiles that are closer to the base station cause significant interference to the mobiles that are further from the station because of non-zero cross-correlation between signature sequences assigned to users. Hence, well-defined power control is essential for proper functioning of the DS-CDMA system.

In general a power control system must first determine the need for power adjustment. This is typically achieved by measuring signal strength, the signal to interference ratio (SIR) or the bit error rate. Open loop control, closed loop control and combinations of the two were proposed. Some schemes propose the use of network wide (centralized) control, while others propose a distributed control mode the adjustment in power may occur in fixed step or adaptively. Reference [9] contains a comprehensive literature review on this subject. In the absence of power control the capacity of the DS-CDMA mobile system is very low. It's necessary to have a dynamic range for control on the order of $80 \mathrm{~dB}$ [9], But it's hard to implement a perfect power control system, because of the following reasons [9],[10]: First, the performance of power control algorithm is affected by the power control error and the dynamic range of transmission. Second, the power control error is caused by the power measurement error, measuring and control signaling take time which result in time delays in the system due to the measurement delay in the power control process. Third, the signals needed for power control have to be estimated and translated to pulses that will take time and cut a part of user bandwidth. Finally, power control systems are complex and integrated. 
Consequently it is very difficult to achieve a perfect power control system; Therefore, in this paper we focus on the effect of imperfect power control on PPIC performance. First, we model a log-linear imperfect power control scenario and evaluate the performance of the PPIC under this scheme to that of the perfect power control scenario. The difference in performance is contrasted using the conventional receiver as a benchmark. We note that the performance enhancement is a function of the partial weights of the PPIC. Therefore, we perform an exhaustive search to find these optimal weights for various signal to noise ratios in order to minimize the probability of error. We compare the results for the optimal weights for the perfect and imperfect power control scenarios. It is shown that the imperfect power control scenario causes a shift in the optimal weights as well as an increased sensitivity of the scheme to deviations from the optimal weights.

The remainder of the paper is organized as follow. Following this introduction, the system model is developed in Section II for the PIC and the PPIC schemes. The numerical results are discussed in Section III, where we detail the layout of the simulations and present a thorough discussion of the results. Finally, Section IV contains conclusions.

\section{SYSTEM MODEL}

Consider a synchronous CDMA system with BPSK modulation for the transmission over an AWGN channel. The transmitted signal for the $i^{\text {th }}$ user can be expressed as: $S_{i}(t)=A_{i} c_{i}(t) \cos \left(\omega t+\theta_{i}\right)$ where $\theta_{i}$ is the carrier phase, $A_{i}=\sqrt{P_{i}} B_{i}(t)$, where $P_{i}$ is the signal power and $B_{i}(t)=\sum_{m=-\infty}^{\infty} a_{i}^{(m)} p\left(t-m T_{h}\right)$ is the data signal, where $a_{i}$ denotes an equiprobable binary sequence taking values \pm 1 and, $p(t)$ is a rectangular pulse with duration $T_{b} . c_{i}(t)$ is the signature waveform.

The received signal $r(t)$ after the demodulation of the composite transmitted signal in AWGN channel can be written as:

$$
\begin{aligned}
r(t) & =\sum_{i=1}^{K} s_{i}(t)+z(t) \\
& =\sum_{i=1}^{K} \sqrt{P i} B i(t) c_{i}(t)+z(t)
\end{aligned}
$$

where $K$ is the number of active users in the cell of CDMA system, $z(t)$ is the additive Gaussian noise with double sided power spectral density No/2.

At the receiver a matched filter is used for despreading, the discrete time output of the matched filter for $i^{\text {th }}$ user, at the correct sampling instant for the $\mathrm{m}^{\text {th }}$ bit is given by: 


$$
\begin{aligned}
y_{i}^{(m)} & =\frac{1}{\sqrt{T b}} \int_{(m-1) T b}^{m T b} r(t) c_{i}{ }^{\circ}(t) d t \\
& =\sqrt{E_{b i}} a_{i}^{(m)}+\sum_{\substack{j=1 \\
j \neq i}}^{K} \sqrt{E_{b j}} a_{j}^{(m)} \rho_{j i}^{(m)}+z_{i}
\end{aligned}
$$

where "*" represents complex conjugate. $E_{b i=P_{i} T_{b}}$ is the bit energy of the $i^{\text {th }}$ user, and $\rho_{j i}$ is the cross correlation ratio between codes, which is given by:

$$
\rho_{j i}^{(m)}=\frac{1}{T_{b}} \int_{(m-1) T b}^{m T b} c_{j}(t) c_{i}^{*}(t) d t, \quad i, j=1,2, \ldots, K
$$

The Gaussian noise component of the $i^{\text {th }}$ user after despreading is expressed as:

$$
z_{i}=\frac{1}{\sqrt{T_{b}}} \int_{(m-1) T_{b}}^{m T h} z(t) c_{i}^{*}(t) d t
$$

Note that the second term at the right hand side of (2) represents the MAI, which is the target of cancellation. For convenience we will drop the time index $m$ in the following discussion to obtain the estimates of data $a_{i}$.

For the conventional receiver (Matched Filter) the decision rule is:

$$
\hat{a}_{i}=\operatorname{sgn}\left\{Y_{i}\right\}
$$

where $\operatorname{sgn}\{$.$\} is the sign function and$

$$
Y_{i}=\operatorname{Re}\left\{y_{i}\right\}
$$

The data estimated by the matched filter contains the MAI caused by other users. Therefore, if a conventional multistage PIC [7], [8] is used to subtract the MAI and assuming the first user is the user of interest we estimate the interference as:

$$
\hat{I}_{1}^{(k)}=\operatorname{Re}\left\{\sum_{i=2}^{K} \sqrt{E_{b i}} \hat{a}_{i}^{(k-1)} \rho_{i 1}\right\}
$$

then the receiver makes the following decision for first user as:

$$
\hat{a}_{1}^{(k)}=\operatorname{sgn}\left\{Y_{1}-\hat{I}_{1}(k)\right\}
$$

This scheme is illustrated in Figure 1.

In (8), the subscript $k$ is for $k^{\text {th }}$ stage of PIC, and $K$ is for the number of users. In PIC, the receiver attempts to completely remove the MAI. Nevertheless, the performance may be worse than that without cancellation if the decision is wrong in the previous 
stage. Therefore Divsalar \& Simon suggest Partial Parallel Interference cancellation (PPIC) [4] using the following formula:

$$
\begin{gathered}
\left.\tilde{a}_{1}^{(k)}=p^{(k)} \mid Y_{1}-\hat{I}_{1}^{(k)}\right]+\left[1-p^{(k)}\right] \tilde{a}_{1}^{(k-1)} \\
\hat{a}_{1}^{(k)}=\operatorname{sgn}\left\{\tilde{a}_{1}^{(k)}\right\}
\end{gathered}
$$

where $p^{(k)}$ is the weight for the kth stage cancellation which is decided by a trial and error computer search. Figure 2 illustrates the PPIC scheme using the weights.

The decisions become more reliable as more interference is removed with the increasing number of stages. Therefore, the partial weights are increased in the successive stages to increase the amount of cancellation. Considering equations (9) and $(10)$ the estimate $\hat{a}_{1}^{(k)}$ is based on the soft output $\widetilde{a}_{1}^{(k)}$ which consists of two terms one is like the conventional PIC but weighted and the other is the soft output of the previous stage.

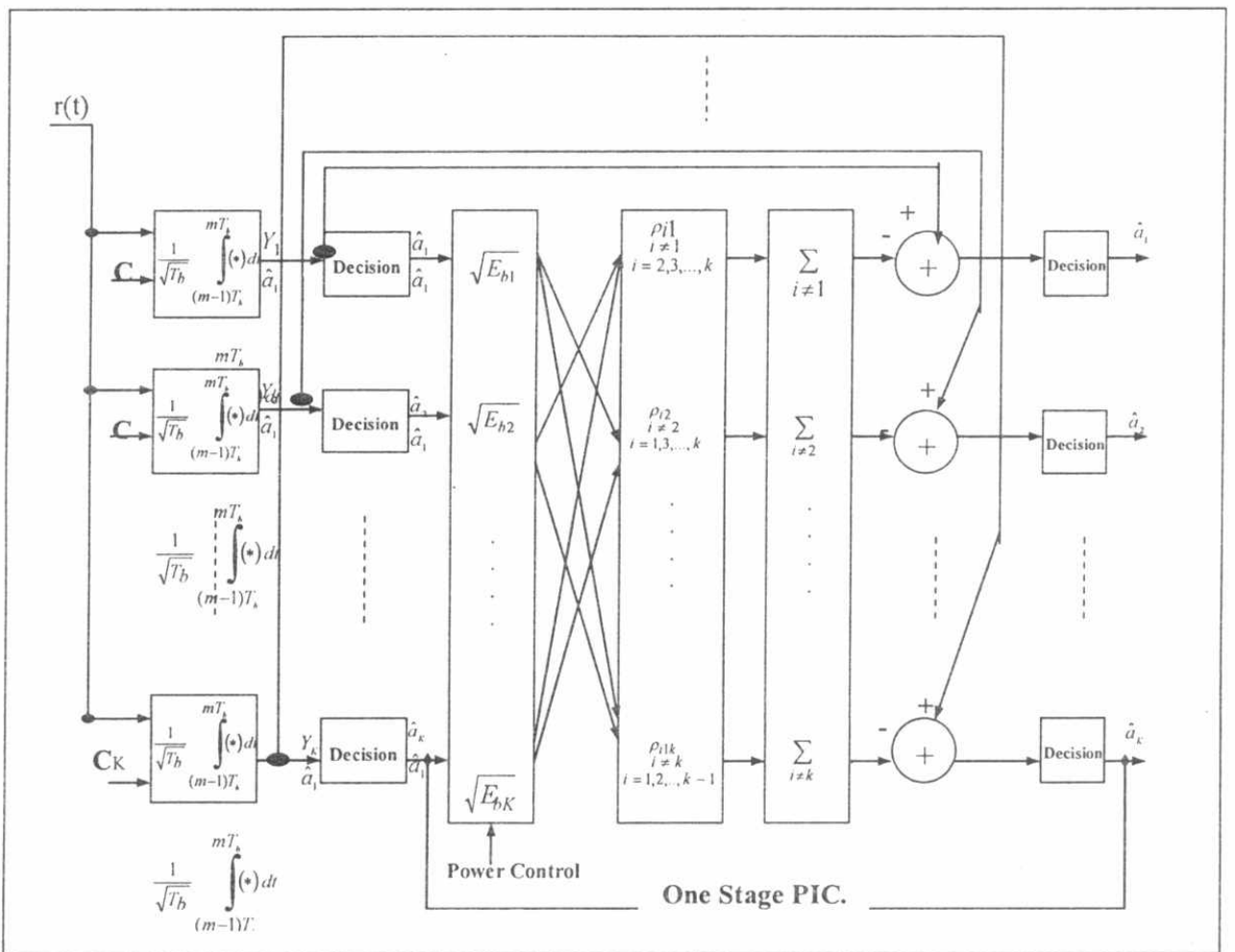

Fig.1. The Conventional Parallel Interference Cancellation Scheme (PIC) 


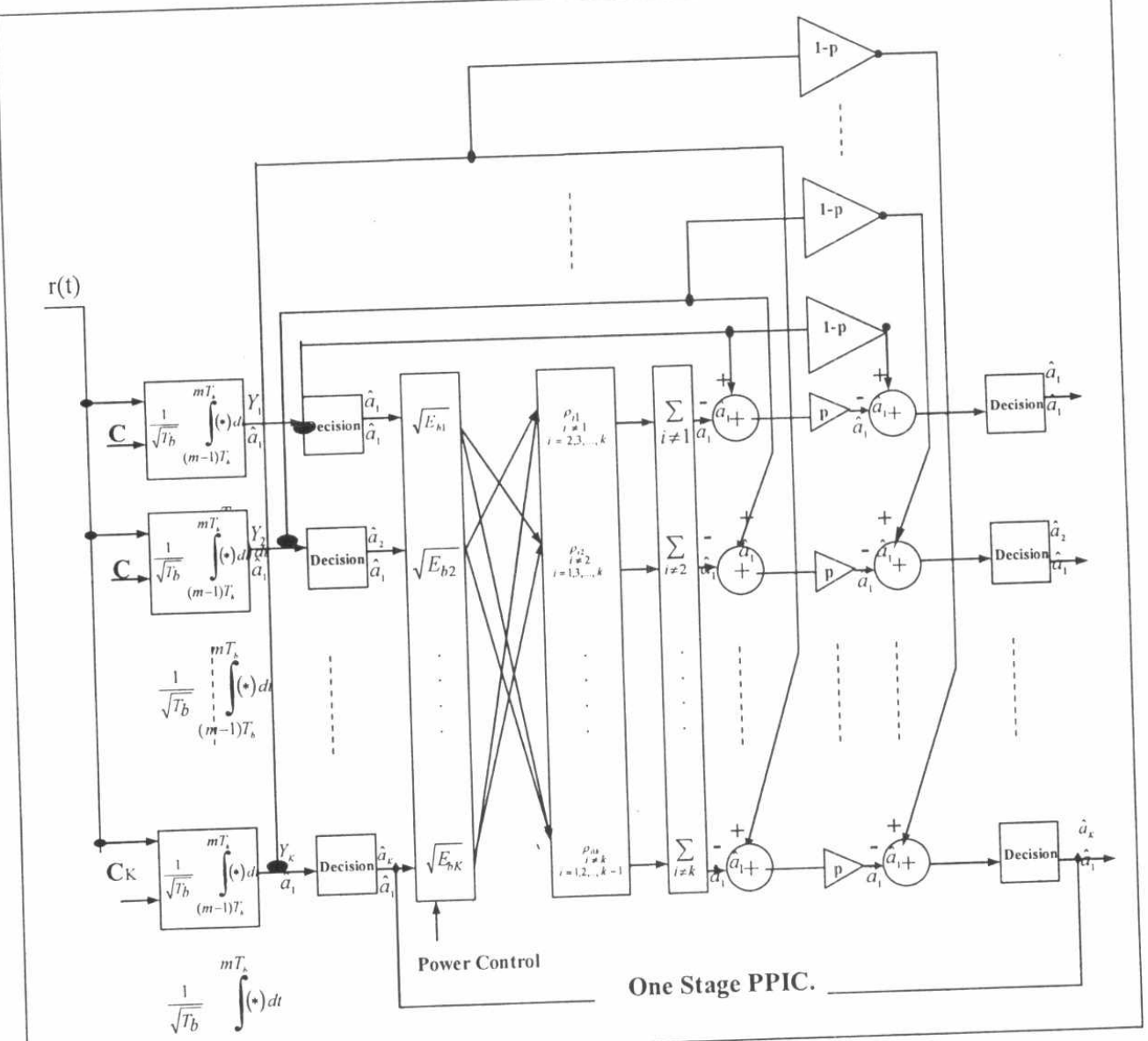

Fig. 2. The Partial Parallel Interference Cancellation Scheme (PPIC).

\section{NUMERICAL RESULTS}

The performance of a PPIC scheme is evaluated using simulations under two power The first scheme represents a perfect power control scheme, where from second the power received from all users is equal at the receiving baser of the users scheme models an imperfect power control scenario, the values of the power in is log-linearly distributed at the receiver. This means that the user with median $\mathrm{dB}$ are linearly increasing. We focus on the performance $12 \mathrm{~dB}$ above and below the power. In particular, we consider the pow median user. This variance is denoted $Q$. 
The multiuser receiver used is a PPIC receiver as modeled by (9) and (10). The system is considered to have a processing gain of 31 (length of the spreading code) and the number of users $\mathrm{K}$ is 31 . Therefore, the system is considered to operate under full load. Unless otherwise noted, $E_{b} / N_{0}$ readings refer to user number 16 with median power.

As a benchmark, two other receivers are simulated and compared to the PPIC performance in the two power control scenarios. The first benchmark receiver is the conventional matched filter receiver modeled in (5). The performance of the conventional receiver is in general worse than the PPIC receiver, except possibly when the PPIC receiver erroneously estimates the interference. The second benchmark receiver is the single user receiver (with no MAI) which is used as a lower bound on PPIC performance.

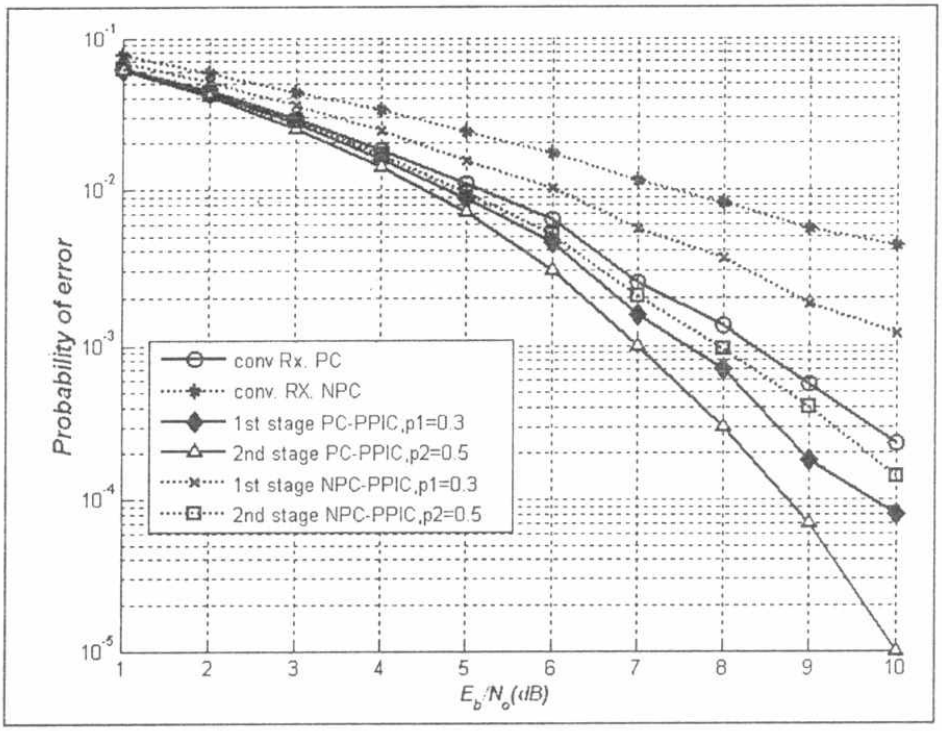

Fig.3. The performance of PPIC for perfect (PC) and imperfect (NPC) power control, AWGN at weights $p 1=0.3, p 2=0.5$ 


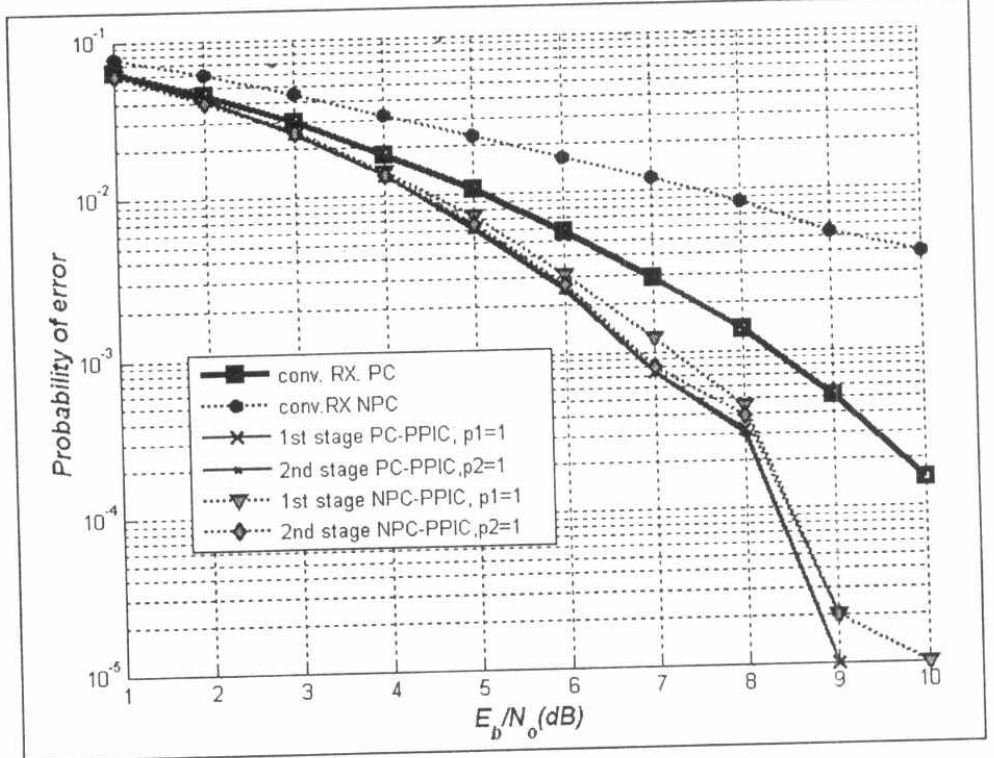

Fig. 4. Performance of PPIC for perfect (PC) and imperfect (NPC) power control, AWGN at weights $p 1=1, p 2=1$

Figure 3 compares the bit error rate performance of a two stage PPIC receiver under the two power control scenarios. For this particular case, the partial cancellation weights are 0.3 and 0.5 for the first and second stages respectively. The effect of the imperfect power control is evident on both the PPIC receiver and the conventional receiver. However, the loss in performance is much less in the PPIC receiver than in the conventional receiver. While the conventional receiver suffers a $1 \mathrm{~dB}$ loss at an error rate of 10-2 and more than $2 \mathrm{~dB}$ loss at 10-3, the PPIC receiver suffers negligible loss at $10-2$ and less than $1.2 \mathrm{~dB}$ at $10-4$. Furthermore, the first stage of the $\mathrm{PPIC}$ is inferior to the conventional receiver under imperfect power control. The second stage removes a substantial amount of interference and minimizes the degradation in performance due to the imperfect power control.

Since the PPIC's efficiency in interference cancellation is affected by the partial weights of each stage, the same scenarios are studied for unity weights in both stages. The results are shown in Figure 4 . It is interesting to note that the interference is almost completely cancelled by the PPIC receiver in the first stage even under imperfect power control. Since we consider an AWGN channel and perfect estimation of the user's energies, it is not surprising that the estimate of the first stage is an accurate representation of the actual interference. It is also worth 
noting that performance is marginally close to the single user lower bound as will be illustrated in Figure 6.

The PPIC scheme with unity weights is in fact the PIC. Here, we attempt to find the optimum weights for the PPIC scheme with an exhaustive search over all weights and over the Eb/No range of interest. For each combination of $p 1$ and Eb/No we evaluate the probability of bit error, and then search for the pair that minimizes this probability of error. One result of such a search is shown in Figure 5 under the perfect power control scheme. It is clear that the minimum probability of error occurs at unity weight. Which means that the single stage PIC is optimum when the estimates for the users are correct.

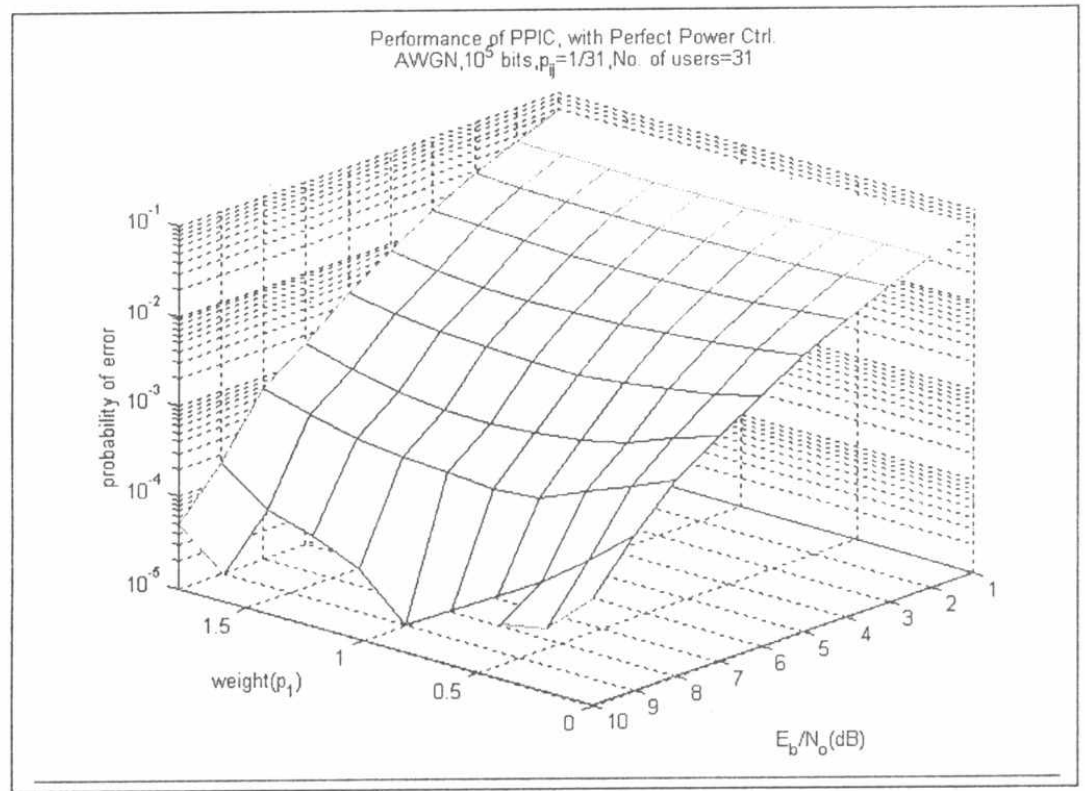

Fig.5. 3D plot for PPIC performance, perfect power control, one stage

Since it is impractical to have a correct estimate of the users' energies at all times, PPIC receivers are usually operated with non-unity weights. Which means that the scenario in Figure 3 is closer to the practical case. Therefore, we simulate the effect of deviation from unity weights (optimum weights) for both power control scheme. The results are shown in Figure 6. 


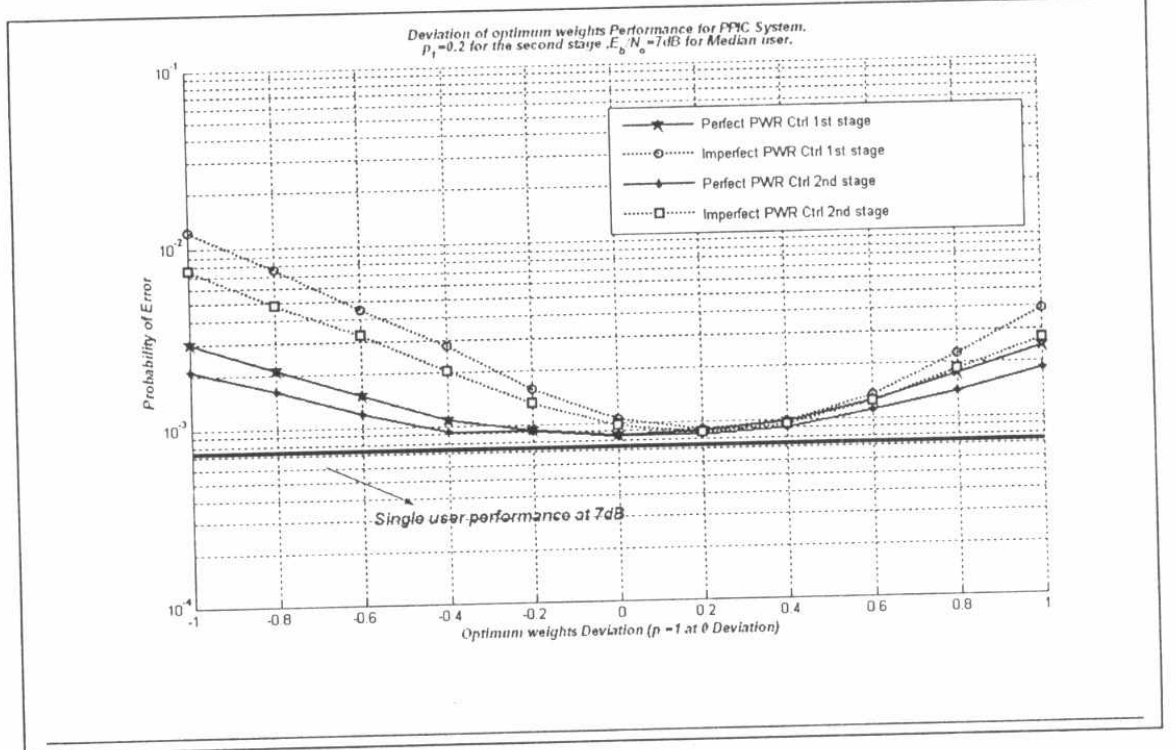

Figure( 6 ): The effect of optimum weights deviation

Figure 6 shows that if the PPIC receiver is operated with weights deviated from the optimum weights, which are unity for the ideal case. It can be noted from the figure, that the imperfect power control scenario results in more susceptibility to loss in bit error performance. The zero point on the $x$-axis corresponds to the partial weight equals one. Two observations can be made from the figure. First, the optimum weights for the PPIC scheme under the imperfect power control scheme is shifted from 1 to 1.2 for both stages. Second, as the weights of the PPIC deviate from their deal values, the imperfect power control schemes suffer more in terms of error performance In the figure, the perfect power control scheme is studied at $E_{b} / N_{0}=7 \mathrm{~dB}$ for all users, this corresponds to the median power in the imperfect power control scheme.

\section{CONCLUSIONS}

The effect of imperfect power control on the performance of the PPIC receiver is studied. It is shown that the PPIC receiver can effectively reduce the effect of imperfect power control compared with other types of receivers. A study of the optimum weights of the PPIC receiver shows that imperfect power control changes the optimum weights that can result in single user performance. Furthermore, imperfect power control causes the receiver to become more susceptible to performance degradation as a result of deviation from optimal weights. Similar 
studies are currently being performed for the fading channel and under more realistic conditions e.g. imperfect estimation of users' energies.

\section{REFERENCES}

[1] G.Xue, J.Weng, T.Le-Ngoc, and S.Tahar, "Adaptive Multistage Parallel Interference Cancellation for CDMA", IEEE J. Select. Areas Commun., vol.17, no.10, pp.1815-1827, Oct. 1999.

[2] S. Verdú, "Multiuser detection", Cambridge university press (C1998

[3] S. Verdú, "Minimum probability of error for asynchronous Gaussian multiple access channels," IEEE Trans. Inform. Theory, vol. IT-32, pp. 85-96, Jan. 1986.

[4] D. Divsalar, M. K. Simon, and D. Raphaeli, "Improved parallel interference cancellation for CDMA," IEEE Trans. Commun., vol. 46, pp.258-268, Feb. 1998.

[5] R. Lupas and S. Verdu, "Linear multiuser detectors for asynchronous code division multiple access channels," IEEE Trans. Inform. Theory, vol. 35, pp. 123136, Jan. 1989

[6] P. Patel and J. Holtzman, "Analysis of a simple successive interference cancellation scheme in a DS/CDMA system, "IEEE J. Select. Areas Commun., vol. 12, pp. 796-807, June 1994.

[7] D. Divsalar and M. K. Simon, "Improved CDMA performance using parallel interference cancellation," JPL Publication 95-21, Oct. 1995.

[8] M. K. Varanasi and B. Aazhang, "Multistage detection in asynchronous code-division multiple-access communications." IEEE Trans. Commun., vol. 38, pp. 509-519, Apr. 1990.

[9] D. M. Novakovic and L. Dukic, "Evolution of the Power Control Techniques for DS-CDMA Toward 3G Wireless Communication Systems," IEEE Commun. Surveys, Available from http://www. comsoc. org/pubs/surveys, $4^{\text {th }}$ Quarter 2000.

[10] W. M. Tam and F. C. M. Lau, "Analysis of Imperfect Power Control in CDMA Cellular Systems," IEEE Trans Comm. PP.892-897, May 1997.

[11] J. G. Proakis, Digital Communications, $4^{\text {th }}$ ed. New York: McGraw-Hill, 2001.

[12] Simon Haykin, "Communication Theory", 3rd edition, John Wiley, 1994. 